INTERNATIONAL DESIGN CONFERENCE - DESIGN 2018

https://doi.org/10.21278/idc.2018.0261

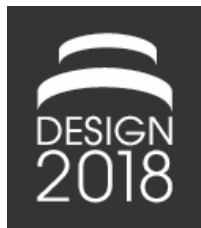

\title{
CREATIVITY UNDER PRESSURE: USING DISTANT SEMANTIC FIELDS FOR FAST ACTIVATION OF DIVERGENT THINKING IN ENGINEERING STUDENTS
}

\author{
A. Beghelli and P. Prieto
}

\begin{abstract}
Creativity is one of the most valued professional skills. However, creativity-training opportunities are scarce in engineering programs. In this paper we present an interventional study for fast activation of divergent thinking in non-trained people. Based on the associative creativity theory, we force the participants to connect semantically distant concepts to generate divergent ideas in a short time. The effect was evaluated using a pre-post test $(n=27)$. We found a significant increase in the number of creative ideas generated after a 2.5 -hour intervention.
\end{abstract}

Keywords: divergent thinking, design creativity, associative creativity theory, human behaviour, design methods

\section{Introduction}

Creativity is one of the most valued professional skills of engineers (Boyett et al., 2010; Tomasco, 2010; Chak, 2011). However, several studies show that engineering students are seldom exposed to practices that foster the various stages of the creative process. Instead they are routinely subject to learning experiences focusing on the technical aspects of engineering that emphasize analytical and convergent thinking (Kazerounian and Foley, 2007; Andersson and Andersson, 2010; Daly et al., 2014; Cropley, 2015).

This paradoxical reality leads to many project-based engineering courses requiring creative solutions from engineering students without any formal training in creativity practice/techniques during their academic life. The results are usually disappointing: engineering students, who usually see themselves as non-creative persons (in line with a study showing that just 3\% of the population associated the concepts "creative" and "engineering", Stouffer et al., 2004), confirm this perception as the generation of novel ideas in domain-specific challenges is very difficult without having exercised the "creativity muscle" enough. Engineering instructors, on the other hand, value creativity training and opportunities (Kazerounian and Foley, 2007) but the emphasis on the technical skills acquisition of engineering programmes hinder the allocation of time for a lengthy creativity-training module. Although preliminary evidence showing effective blending of creativity-oriented design methods and technical content in an engineering course has been reported in (Beghelli and Prieto, 2015), further research on advanced courses is still required. Given this situation, an increasingly common request to the authors of this paper is that of a "fast creativity training", meaning 1 to 2 class sessions at the beginning of the academic term to prepare the students for the forthcoming project.

Faced with the challenge of strengthening the "creativity muscle" of non-trained students in just a couple of hours, we devised an interventional study focused on the quick activation of divergent thinking with 
the aim of producing creative ideas. The study focused on training students on 3 specific idea generation methods. The reasons behind this decision are as follows:

- Practising divergent thinking, "the capacity to generate multiple alternative solutions as opposed to the one correct solution" (Scott et al., 2004), is a must as this skill is usually the least represented in typical engineering courses (Daly et al., 2014) in spite of being a key aspect of creative thought.

- Previous studies have shown that teaching ideational skills can have the same effect on the overall scores of creativity tests that a longer training (Clapham, 1997). Given our strict time constraints, we focused on the activity with demonstrated highest impact.

- Among the many different idea generation methods, we selected 3 that force the students to connect very distant concepts in their associative networks. By doing so we build on the associative basis of the creative process stated by (Mednick, 1962) and "guarantee" the generation of "out of the box" ideas. However, this is a risky decision as the cognitive effort of connecting apparently unrelated concepts is very high, specially for non-trained people that could transit to a blank state: Do you want me to design a clock that behaves like a Ed Sheeran song? No sense! Therefore, providing a step-by-step methodology for the student to navigate his/her associative network is key to succeed in making the distant connection so the cognitive resources of the participants are focused on the creativity task and not on complementary or preliminary steps (Chandler and Sweller, 1991).

We carried out a pre/post test $(n=27)$ to verify whether the designed intervention was effective in providing the ability to quickly generate creative ideas to engineering students without formal training in creativity methods.

In the rest of this paper we present a brief review of the area of creativity and associative networks to then describe the interventional study. Next we present results in terms of the number of ideas (classified as non-creative or creative) generated by the 27 engineering students before and after a 2.5 -hour (approximately) session. Finally, we conclude the paper.

\section{Creativity and associative networks}

A semantic field is a group of words semantically related covering a certain conceptual domain (Kittay and Lehrer, 1981; Hills et al., 2015). For example, the words run, walk, skip, stride all refer to motion and thus, they belong to the same semantic field (Clark, 1972). Different semantic fields are in turn connected among them, forming a semantic or associative network. In such a network every node represents a word or a concept and related nodes are connected with a direct link. Through life, every person builds his/her own associative network based on his/her experiences. Thus, whilst someone could associate the word hammer with love because her beloved grandfather was a carpenter another could associate hammer with pain, as he lost one finger during an accident involving a hammer. In Figure 1 a graph representation of two semantic fields belonging to a much larger semantic network of 96 concepts is shown (the example has been extracted from Kenett et al., 2014).

In 1962 Sarnoff Mednick outlined an associative theory of creative thinking (Mednick, 1962): “we [...] define the creative thinking process as the forming of associative elements into new combinations [...]. The more mutually remote the elements of the new combination, the more creative the [...] solution." That is, according to Mednick, creative solutions are those connecting concepts belonging to very distant semantic fields. In the example of Figure 1, that would be the case of an idea connecting the concepts of stapler and basketball. In such a network those concepts are at a semantic distance of at least 10 links. Mednick also hypothesized that highly creative individuals would exhibit a more connected associative network that would allow them to arrive more easily to less likely associations.

The hypothesis of Mednick has been later confirmed by several studies. For example, in (Durso et al., 1994) it was shown that people who were able to solve a given problem had a semantic network equipped with key links that non-solvers did not have; Rossman and Fink demonstrated that people identified as highly creative estimated the distance between unrelated word pairs as lower than people with low levels of creativity (Rossman and Fink, 2010); in a similar line of research in (Kenett et al., 2014) it was shown that a group of people with low creativity ability exhibit a network with a lower number of connections 
resulting in longer distances between concepts whilst in (Benedek et al., 2017) the same conclusion was drawn at the individual level.

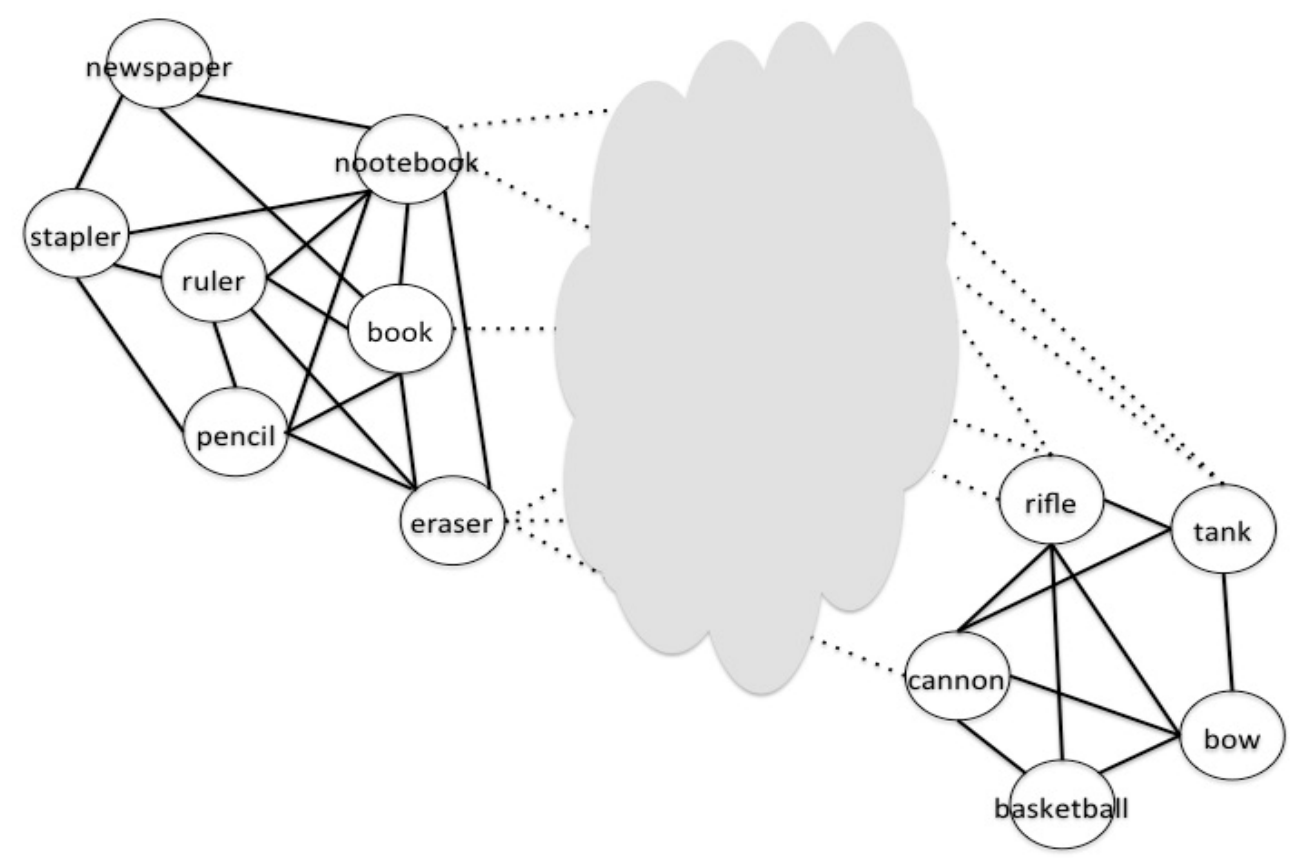

Figure 1. Visualization example of two semantic fields in an associative network of 96 concepts (extracted from Kenett et al., 2014)

Based on these findings, we propose an interventional study that forces subjects non-trained in creativity techniques to make connections of different concepts belonging to distant semantic fields in a short time. In this way, we expect the subjects to move from few non-creative ideas to a significant amount of outof-the-box solutions. The interventional study is described in the next section.

\section{Interventional study}

The method used to force students to quickly generate creative ideas by connecting distant concepts is made of the following 4 steps:

\subsection{Partial view of the subject's semantic fields}

Subjects are asked to write down their semantic fields for the concepts related to the creativity task. For example, if the task is "new uses for a hammer", the subjects must list all words they freely associate to the word hammer. By doing so, and following Mednick's definition of creative ideas, it is possible evaluating which of the ideas generated later by the participant are not creative: an idea is not creative if connects concepts belonging to the immediate semantic field of a subject. For example, if the word "smash" is in the semantic field of hammer, then the idea of "using a hammer to smash ice cubes" is not a creative idea.

\subsection{Pre-test: Idea generation}

Participants are asked to write as many ideas as they can for the creativity task. There is no time limit for this activity. When the person feels he/she has exhausted the pool of ideas, he/she stops and registers the time. This step allows to quickly classify the level of creativity of the participant based on the number and type of ideas generated in this step: subjects with low levels of creativity should generate a low number of ideas using concepts belonging to the semantic fields visualised in the previous step. To 
obtain an objective classification of whether an idea belongs or not to the semantic field of a person, we apply the following 3 criteria:

- The idea is made of at least one word present in the semantic field of the subject;

- The idea is made of at least one synonym of one word present in the semantic field of the subject;

- The idea is made of at least one word derived from one word (or synonym) present in the semantic field of the subject (e.g. recyclable is derived from recycle).

If an idea meets at least one of these criteria, the idea is classified as non-creative. Table 1 shows the algorithm used to perform the classification. In this way, only one person is required to perform the classification, as the algorithm does not resort to subjective criteria.

Table 1. Algorithm used to classify ideas as non-creative or creative

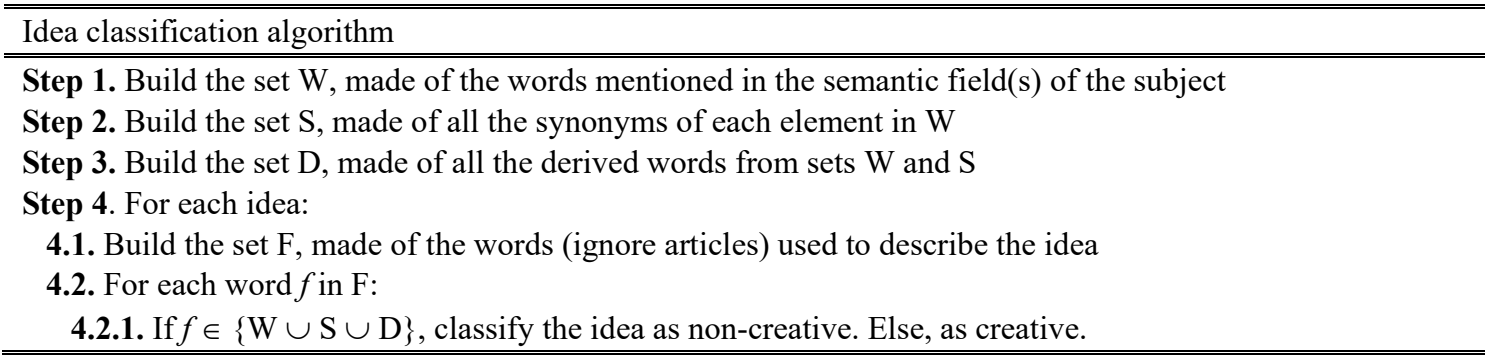

To build the sets S and D the websites wordreference.com/sinonimos (in Spanish) and gramaticas.net (->Morfología -> Derivación) were used, respectively.

\subsection{Introduction to creativity and associative networks}

Subjects are briefly lectured about: a) the 4-step creative process, b) the associative creativity theory, c) the definition and examples of semantic fields/networks and d) how the connection of close/distant semantic fields leads to low/high creative ideas. This introduction takes no more than 15 minutes. Its purpose is for the untrained engineering students to understand why they are applying such unfamiliar/odd techniques and avoid inhibiting unfeasible or absurd ideas.

\subsection{Post-test: Idea generation from distant concepts}

Subjects are presented with 3 methods to generate ideas and examples on how to apply them. Among the many idea generation techniques, we selected 3 methods that force the participants to connect distant concepts out of their immediate semantic field. The distant concepts are provided to the participants by the same methods. Doing these unusual associations represents a very difficult challenge for non-trained people because a direct connection does not necessarily exist in their semantic network and it is difficult for them to find/build a "path" from the concept associated to the creativity task to the distant concept used as an analogy. Thus, every method is equipped with a step-by-step application of it instead of just describing how to apply them, the usual approach for students already trained in creative work. The 3 methods are as follows:

\subsubsection{Storytelling}

The subject must choose a children tale, movie or any other story that appeals to him/her. From a semantic network perspective, the story will contain many concepts with a long semantic distance from the main concept(s) of their creativity task. It is important that the person knows the story well, as the cognitive effort must be focused in finding unusual connections and not in creating/remembering the story (Chandler and Sweller, 1991). Next, s/he has to tell this story changing the characters or the objects in it into the main concepts of his/her creativity task and write down all the associations that come up spontaneously by doing this. To facilitate this task, we provide a short leaflet with the instructions, an example and a very well known story ready to be filled with concepts. Table 2 shows an extract of one of the leaflets: 
Table 2. Example of a leaflet supporting the storytelling technique

INSTRUCTIONS

Use the blank spaces to write down a main concept related to your creativity challenge. By doing so, you will think of some unusual associations between your creativity challenge concept and a concept of the story. Register them, no matter how unfeasible or absurd they can seem at first.

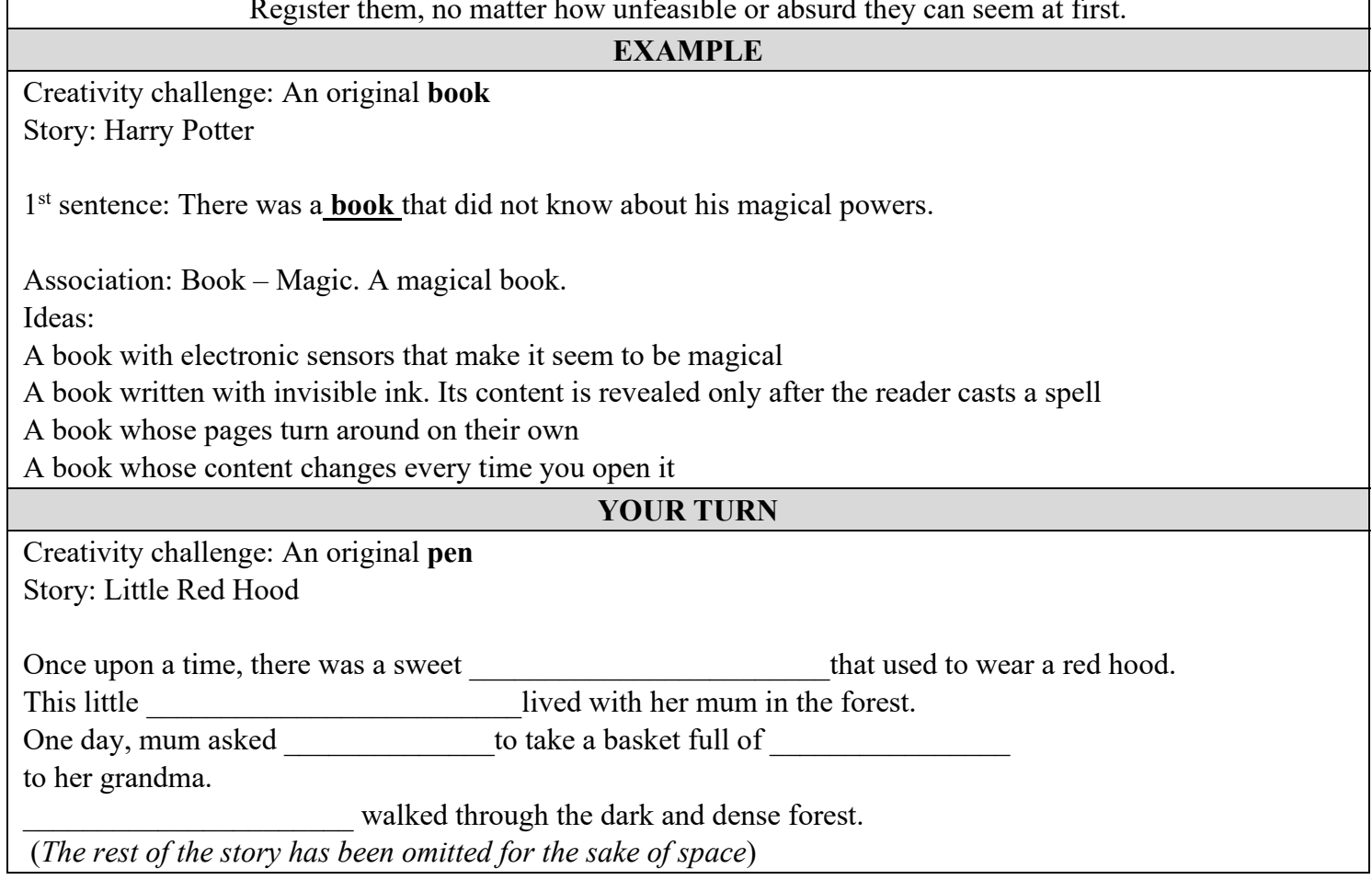

\subsubsection{Metaphors}

A metaphor "involves the bringing together of two [...] conceptually incongruous domains" (Kittay and Lehrer, 1981) and thus, it is a potential source of highly creative ideas. Because of this, the use of metaphors is very well known in creativity-intensive professions. To use metaphors we first ask the subjects to select 3 different types of objects from a pre-built list including pieces of art, animals and everyday objects. To facilitate the connection between the distant concepts, the subjects must list the words they associate to the selected object (the semantic field of the object) and think whether they can solve the creativity challenge by transferring one of these associations as a characteristic of the original concept. If this is not successful, they can continue expanding the semantic field with $2^{\text {nd }}$ level associations and so on. In this way, they "build a path" (in their associative network) from the object to the main concepts of the creativity task. The following is an extract of ideas generated by one student during an ideation session using this technique:

- Creativity challenge: new uses for a hammer;

- Object selected from the list: perfume;

- Concepts in the semantic field of perfume: smell, glamour, spray, flask;

- New ideas: hammer as a smell-stamping device, a luxury hammer, use the hammer as a perfume applicator by adding a soft material in the head, glass hammer to store perfume.

\subsubsection{Opposite concepts}

The subject must write down the antonyms of selected words in the semantic field of the key concepts of the creativity challenge. By doing so, we expect to force the person to think on very unusual functions or appearance aspects for the object of the creativity task. The following is an extract of ideas generated by one student during an ideation session using this technique:

- Creativity challenge: new uses for a hammer; 
- Concepts in the semantic field of hammer: strike, hard, nail;

- Antonyms: stroke, soft, unlock;

- New ideas: a hammer equipped with softfluffy material at the head so you can use it to pet/stroke/massage and hold your head while napping, a hammer as a door holder.

\section{Results}

We carried out the intervention with 27 students in their $3^{\text {rd }}$ year of Business and Administration Engineering ( $30 \%$ female), without any previous formal training in creativity methods. A local company looking for novel ideas for a "sustainable shoe polish" defined the creative challenge. The instructor of the course gave us the usual meeting time of the course for that week: two consecutive 70-minute blocks with a break of 20 minutes in between. The first 70 minutes were devoted to present the goal of the session, to write down the semantic fields of the concepts "shoe polish" and "sustainability"; generate the first set of ideas; the brief lecture and introduce/apply the first method. Then, a break of 20 minutes was given. The second block was devoted to generate ideas using the remaining described methods. The results obtained after applying the methodology are presented in the following.

\subsection{Subject's semantic fields}

Students were asked to write all the concepts they associated to the concepts of "shoe polish" and "sustainability". Ten minutes were allowed to complete this step. There were a total of 194 different associations generated for the concept "shoe polish" with a mean value of 18 associations per person. For the concept "sustainability" 159 associations were generated with a mean of 13 associations per person. Figure 2 shows the frequency histogram of the number of concepts associated to "shoe polish" (left) and "sustainability" (right). For comparison purposes, the number and width of bins of the data for sustainability was used for both histograms. The bin width was chosen according to the rules for quantitative data sets discussed in (McClave and Benson, 1988).
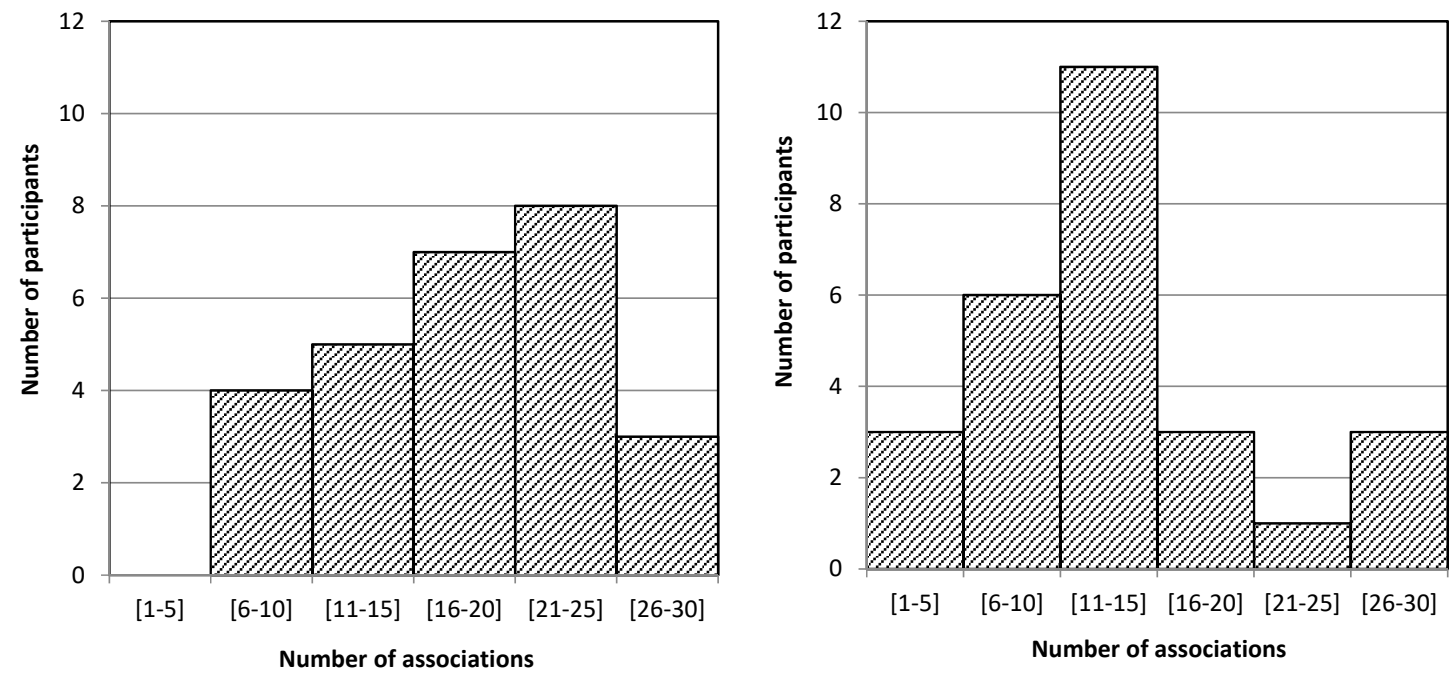

Figure 2. Frequency histogram of the number of concepts associated to "shoe polish" (left) and "sustainability" (right)

Table 3 lists the 10 most mentioned concepts associated to the words "shoe polish". In the table, the first column (from left to right) shows the associated concept, the second column the number of subjects that mentioned that association and the third column shows the type of association: physical attribute (shape, colour, smell, etc.), functionality (mainly verbs), unintended functionality (a functionality the object was not originally devised to have) and interaction object (an object that must be interacted with to perform the function). These categories of associations were identified by means of a bottom-up analysis of the answers provided by the participants. 
It can be seen that the most mentioned concepts are all related to the physical aspect of the object "shoe polish", its functionality (either intended or not) and objects that must be interacted with, to perform the functionality. In fact, out of the total 494 associations made by the subjects, 164 (33\%) corresponded to physical attributes, $93(19 \%)$ to functionality and $64(13 \%)$ to interaction aspects. Thus, it is expected that the non-creative ideas are those related to the physical and functional/interaction aspects of the object as they are in the semantic field of the subjects.

Table 3. 10 most mentioned associations for the concept "shoe polish"

\begin{tabular}{|c|c|c|}
\hline \hline Associated concept & Frequency & Type of association \\
\hline \hline Smell/Smelly/Strong smell & 20 & Physical attribute \\
\hline Clean/Cleaning/Cleaner & 18 & Functionality \\
\hline Polish/Polished & 14 & Functionality \\
\hline Stain/Smudge & 14 & Unintended functionality \\
\hline Dough/Doughy & 13 & Physical attribute \\
\hline Black & 13 & Physical attribute \\
\hline Brightness/Sparkly/Brilliance & 13 & Functionality \\
\hline Brush & 13 & Interaction \\
\hline Metallic container/Metallic box/Tin & 12 & Physical attribute \\
\hline Shoe/Shoes & 12 & Interaction \\
\hline
\end{tabular}

Table 4 shows the same information for the "sustainability" concept. The list was extended to 16 different associations as there were many associations mentioned 6 times. In this case, most associations are related to mechanisms that can be used to provide sustainability, attributes of sustainability and organisations (social, biological, economic, etc.) that are relevant to the concept.

Table 4. 10 most mentioned associations for the concept "sustainability"

\begin{tabular}{|c|c|c|}
\hline \hline Associated concept & Frequency & Type of association \\
\hline \hline Re-use/Recycle/Returnable & 20 & Enabling mechanism \\
\hline Green/Ecological/Eco-Friendly & 20 & Attribute \\
\hline Environment/Nature/Pro-Environment & 19 & Relevant actor \\
\hline Durable/Enduring/Long-term & 12 & Attribute \\
\hline Responsibility/Social Responsibility & 10 & Enabling mechanism \\
\hline Resource saving/Optimization/Efficiency & 9 & Enabling mechanism \\
\hline Biodegradable & 7 & Enabling mechanism \\
\hline Care/Protection & 6 & Enabling mechanism \\
\hline Friendly & 6 & Attribute \\
\hline Companies & 6 & Relevant actor \\
\hline Awareness/Lack of awareness & 6 & Enabling mechanism/Cause \\
\hline No pollution/Pollution & 6 & Enabling mechanism/Cause \\
\hline Innovation \& Entrepreneurship & 6 & Enabling mechanism \\
\hline Clean/Cleanliness & 6 & Enabling mechanism \\
\hline
\end{tabular}

\subsection{Pre-test: Idea generation}

Students were given unlimited time to generate as many ideas as they could to produce a "sustainable shoe polish". They produced a total of 227 ideas, with an average of 8.4 ideas per participant in a mean time of 5.7 minutes. Figure 3 shows the frequency histogram of the time devoted by the participants to generate ideas (left) and the average number of ideas generated for the participants as a function of the time they devoted to such activity (right).

From the figure it can be seen that: 
- Just $20 \%$ participants devoted more than 8 minutes to the activity of generating ideas.

- The number of ideas increased with the time devoted to generate them. It is unclear whether this is an indicator of fluency (more time was allocated to this task because a high number of ideas were naturally generated) or persistence at the task (a high number of ideas was generated because after exhausting a "pool of ideas", more time was dedicated to find a new pool).
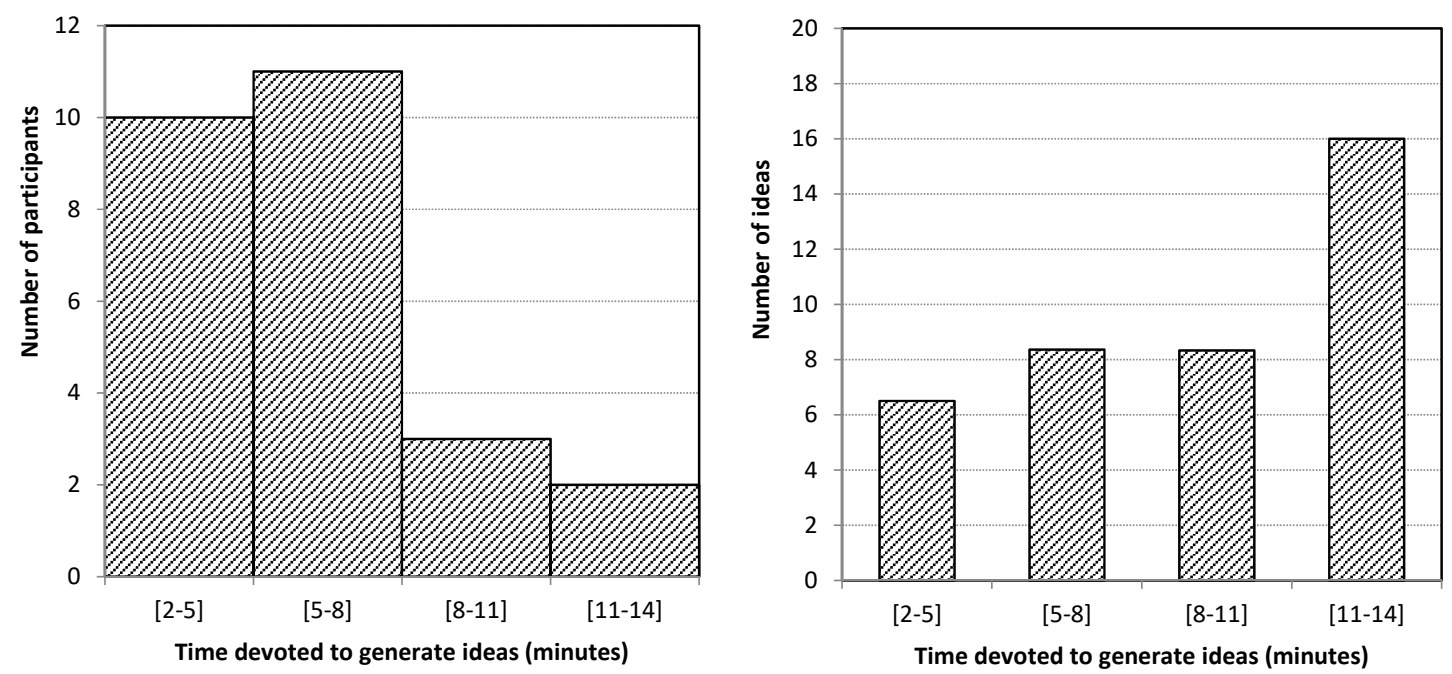

Figure 3. Frequency histogram of time devoted to generate ideas (left) and average number of ideas generated by participants as a function of the time devoted to the activity (right)

In a first attempt, the ideas generated in this stage were classified as non creative or creative depending on whether they were directly derived from the individual semantic fields associated to the concepts "shoe polish" and "sustainability" or not, according to the 3 criteria presented in the previous section. For example, Subject 1 associated the word container to shoe polish and the word recyclable to the concept of sustainability. His first idea: a recyclable container is classified as non-creative as this idea is made of words present in his semantic fields for shoe polish and sustainability.

After using these criteria for classifying ideas we found out that $43 \%$ of ideas were categorized as noncreative and $57 \%$ as creative. However, many ideas classified as creative with this method were extremely common. For example, several subjects did not mention the word biodegradable in their semantic field for the concept sustainability. Therefore, their idea of using a biodegradable content should be classified as creative, although such idea is obviously made of concepts that should be in the semantic field of sustainability. This happened because although a subject did not mention a word in his/her list of associations to a given concept, such word is part of the "collective semantic field" associated to the concept and very likely to be mentioned by the person if more time would have been allocated to the task of listing the associations.

This situation occurred very often and it highlighted the fact that our method to visualize parts of the individual semantic field of a person was not reliable as several obvious associations were left out of the list. We do not know whether this was due to a lack of time or other factors. Future research on fast reliable mechanisms for visualization of the semantic fields of a person is required.

To circumvent this problem, we re-classified ideas using the same 3 criteria, but instead of using the individual semantic field of a subject, we used the collective semantic field made of all the associations generated by the whole group. In that case, the percentage of non creative ideas increased to $74 \%$ and just $26 \%$ can be considered as creative, with a mean of 2.1 creative ideas per subject. Among the 27 subjects, only 6 of them presented 3 or more creative ideas. Those 6 subjects were also subjects belonging to the group that generated more ideas than average.

Examples of non creative ideas generated in this stage are: "biodegradable container/content" (mentioned by 13 subjects), "recyclable container" (mentioned by 9 subjects) and "organic content" 
(mentioned by 6 subjects). Examples of ideas classified as creative are: "content made of leaves", "containers made of peanut shells" and "humid towels that mix with water".

Figure 4 shows the total number of ideas (left) and the number of creative ideas (right) generated in this stage (before the intervention) as a function of the number of associations generated by the participants during the semantic field visualization stage. For example, the set of participants that generated between 12 and 27 associations when building their semantic fields produced and average of 7.1 ideas in this stage (left graph). From them, 1.6 were classified as creative (right graph). For comparison purposes, the scales of the vertical axis of both graphs are the same. It can be seen that the Mednick hypothesis cannot be confirmed when looking at the total number of ideas: the participants with a higher number of associations did not generate the higher number of ideas. However, this pool of ideas has a mixture of creative and non-creative ideas. When we isolate the creative ideas generated, in agreement with the hypothesis of Mednick, subjects with higher number of associations were also the subjects that generated a higher number of creative ideas.
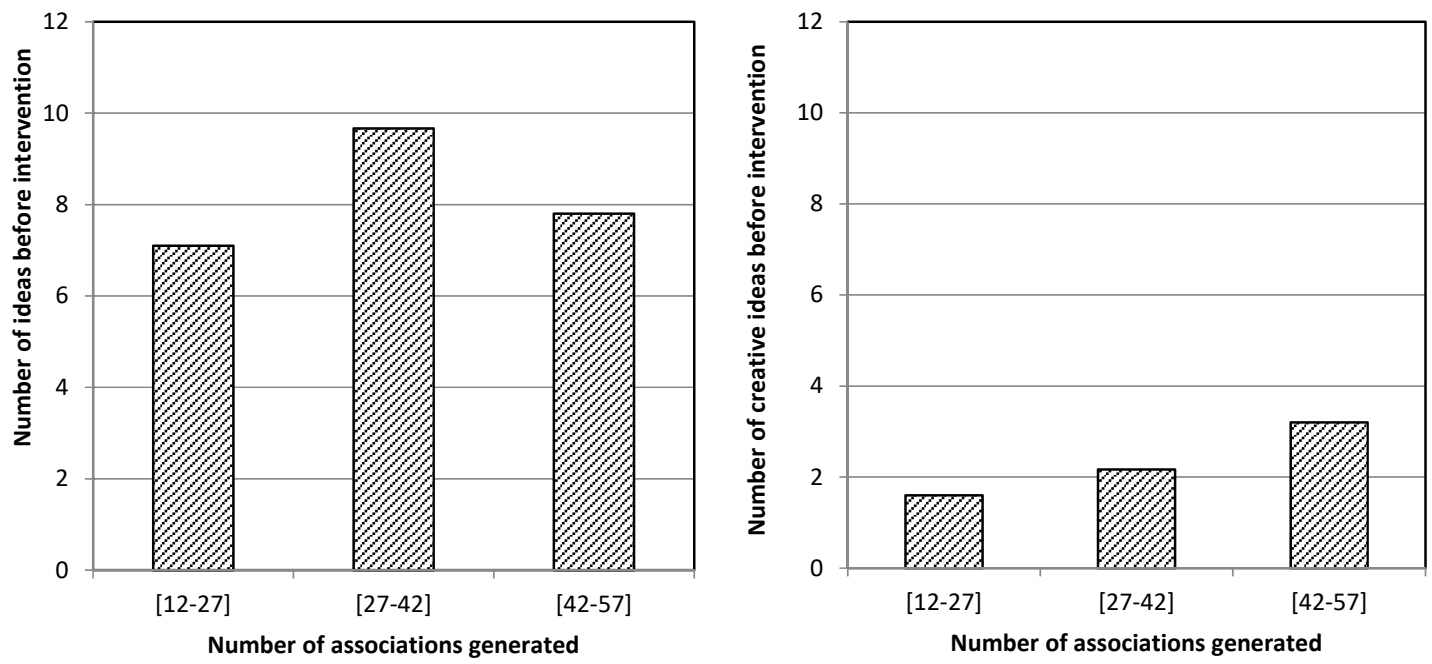

Figure 4. Number of total ideas (left) and creative ideas (right) generated before the intervention as a function of the number of associations generated by the participants

\subsection{Post-test: Idea generation from distant concepts}

Figure 5 shows the total number of ideas generated before and after the intervention for the group (left) and individually (right), highlighting the significant increase in the production of ideas after the intervention. The number of ideas generated by the 27 subjects after the intervention increased to 903 new ideas, with a mean of 33.4 ideas per subject, highlighting the impact of ideation methods on the divergent thinking of non-trained subjects.

After applying the 3 criteria to classify the ideas as non creative or creative (using the collective semantic field), the percentage of creative ideas increased to $73 \%$ (as opposed to just $26 \%$ before the intervention) with a mean of 24.3 creative ideas per subject (i.e. ideas out of his/her immediate semantic field). That is, whilst the number of total ideas increased 4 times after the intervention, the number of creative ideas increased in a factor of 11, highlighting the impact of these methods in generating ideas connecting concepts outside from the immediate semantic field of subjects.

The high number of ideas classified as creative confirms that the "scaffolding" provided to the students to navigate through their semantic networks to find connections between distant concepts was successful. Examples of ideas classified as creative in this stage are: "fragmented small pieces of shoe polish [dough] of different colours to resemble a Britto artwork", "shoe polish as collectables with different shapes and sizes that can be assembled after use to build different toys", "[dough] shoe polish with different colours and shapes of letters that can be used to do wall art in the city", "drop the 
container, a shoe polish that can be applied directly (as a soap)", "a shoe polish with the shape of a book, to offer different colours in the same container".
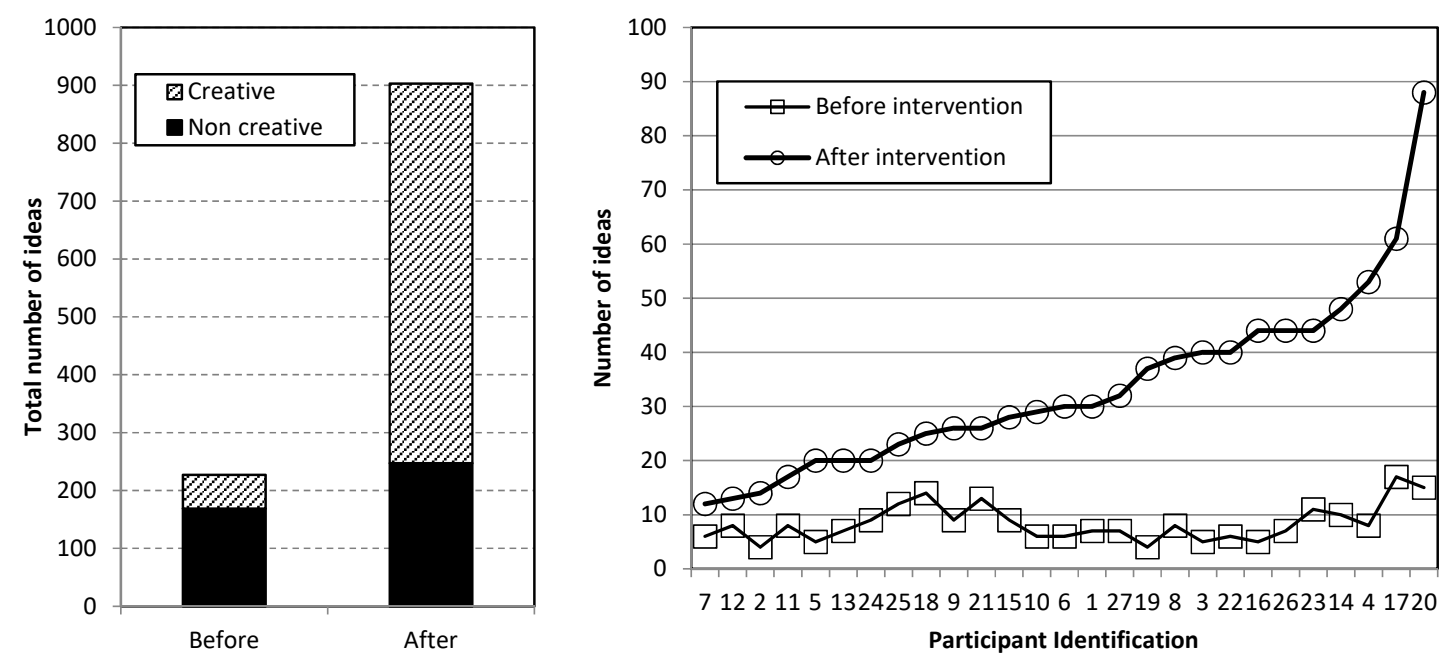

Figure 5. Number of ideas generated by the group (left) and individually (right) before and after the intervention

Finally, analogously to Figure 4, Figure 6 shows the total number of ideas (left) and the number of creative ideas (right) generated after the intervention as a function of the number of associations generated by the participants during the semantic field visualization stage.

After the intervention, the hypothesis of Mednick holds for both cases: the higher the number of associations, the higher the total number of ideas and the total number of creative ideas generated by the participants. It seems that, as participants are pushed beyond the comfortable boundaries of their semantic fields, the individuals with a higher number of connections (associations) exploit this condition and are able to produce a higher number of creative ideas.
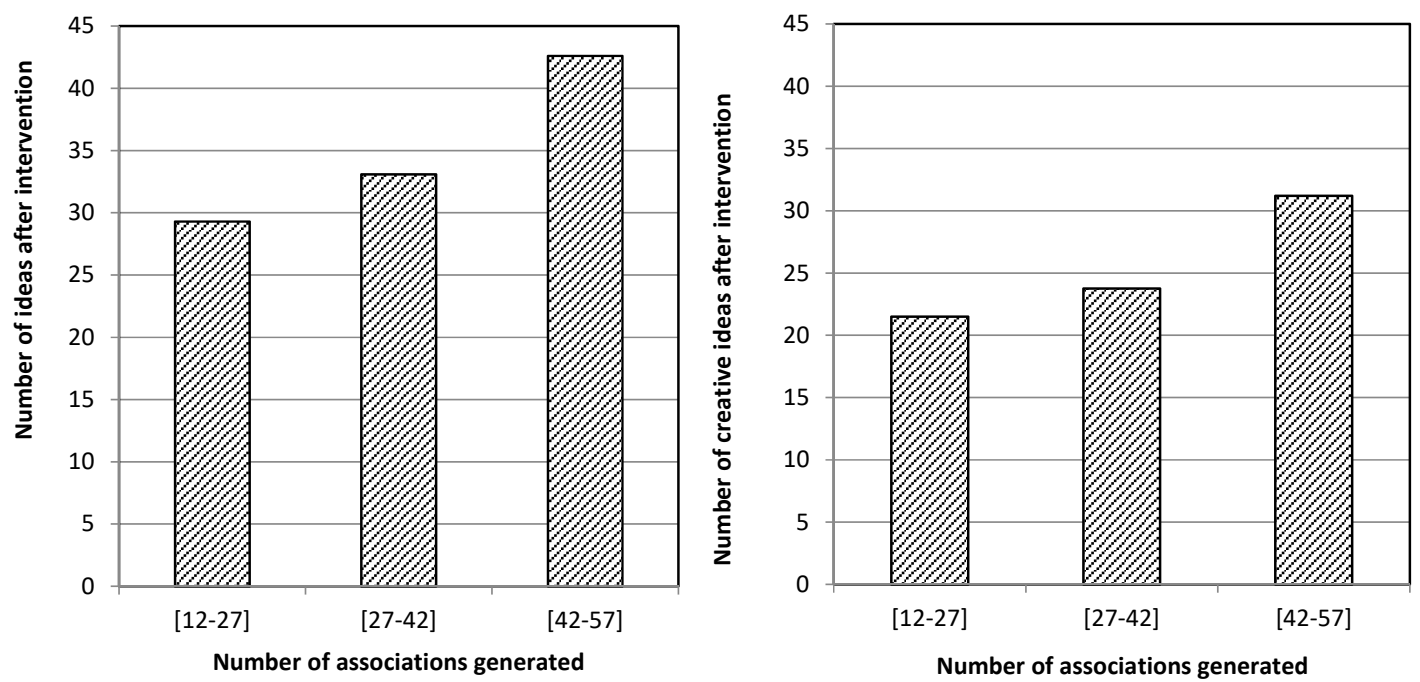

Figure 6. Number of total ideas (left) and creative ideas (right) generated after the intervention as a function of the number of associations generated by the participants

\section{Conclusions}

In this paper we presented an interventional study based on Mednick's associative theory creativity to help non-trained subjects to quickly enter into divergent thinking and generate a high number of "out of 
the box" ideas. To do so, subjects are forced to connect semantically distant concepts. In spite of the high cognitive effort of doing so and the short time available, the intervention proved to be effective: the number of ideas generated increased from a total of 227 (before applying the ideation techniques) to 903 (after applying the ideation techniques) whilst the percentage of ideas classified as creative also increased from $26 \%$ to $73 \%$.

Further research should focus on dealing with the detected limitations of the intervention: Firstly, improving the method to quickly and effectively visualize a complete semantic field of a subject, as it was clear that many subjects forget to mention important associations. Second, comparing the impact of the techniques studied here with other that do not force a connection with very distant concepts would be necessary to quantify the impact of these 3 specific techniques regarding the generation of creative ideas.

\section{Acknowledgements}

Financial support from the Advanced Center for Electrical and Electronic Engineering, Basal Project FB-008 (Conicyt) is gratefully acknowledged.

\section{References}

Andersson, N. and Andersson, P.H. (2010), "Teaching professional engineering skills-industrial participation in realistic role play simulation", Proceedings of the 6th International CDIO Conference / Making Change Last: Sustaining and Globalizing Engineering Educational Reform, Montréal, Canada, June 15-18, 2010, École Polytechnique, Montréal, Canada.

Beghelli, A. and Prieto, P. (2015), "The impact of design methods on the creativity of 1st-year engineering student projects: the case of Computer Programming", Proceedings of the 20th International Conference on Engineering Design (ICED '15), Vol. 8: Innovation and Creativity, Milan, Italy, July 27-30, 2015, The Design Society, Glasgow, pp. 279-288.

Benedek, M., Kenett, Y.N., Umdasch, K., Anaki, D., Faust, M. and Neubauer, A.C. (2017), "How semantic memory structure and intelligence contribute to creative thought: a network science approach", Thinking \& Reasoning, Vol. 23 No 2, pp. 158-183. https://doi.org/10.1080/13546783.2016.1278034

Boyett, K., Courter, S., McGlamery, T., Nathans-Kelly, T. and Nicometo, C. (2010), "Understanding engineering work and identity: a cross-case analysis of engineers within six firms", Engineering Studies, Vol. 2 No. 3, pp. 153-174. https://doi.org/10.1080/19378629.2010.519772

Chak, C. (2011), "Changing engineering curriculum in the globalizing world", New Horizons in Education, Vol. 59 No. 3, pp. 59-70.

Chandler, P. and Sweller, J. (1991), "Cognitive Load Theory and the Format of Instruction”, Cognition and Instruction, Vol.8 No. 4, pp. 293-332. https://doi.org/10.1207/s1532690xci0804_2

Clapham, M.M. (1997), "Ideational Skills Training: A Key Element in Creativity Training Programs", Creativity Research Journal, Vol. 10 No. 1, pp. 33-44. https://doi.org/10.1207/s15326934crj1001_4

Clark, E.V. (1972), "On the child's acquisition of antonyms in two semantic fields", Journal of Verbal Learning and Verbal Behaviour, Vol. 11 No. 6, pp. 750-758. https://doi.org/10.1016/S0022-5371(72)80009-4

Cropley, D.H. (2015), "Promoting Creativity and Innovation in Engineering Education", Psychology of the Aesthetics, Creativity and the Arts, Vol. 9 No. 2, pp. 161-171. https://doi.org/10.1037/aca0000008

Daly, S.R., Mosyjowski, E.A. and Seifert, C.M. (2014), “Teaching creativity in engineering courses", Journal of Engineering Education, Vol. 103 No. 3, pp. 417-449. https://doi.org/10.1002/jee.20048

Durso, F.T., Rea, C.B. and Dayton, T. (1994), "Graph-theoretic confirmation of restructuring during insight", Psychological Science, Vol. 5 No. 2, pp. 94-98. https://doi.org/10.1111/j.1467-9280.1994.tb00637.x

Hills, T.T., Todd, P.M. and Jones, M.N. (2015), "Foraging in Semantic Fields: How We Search Through Memory", Topics in Cognitive Science, Vol. 7 No. 3, pp. 1-22. https://doi.org/10.1111/tops.12151

Kazerounian, K. and Foley, S. (2007), "Barriers to creativity in engineering education: a study of instructors and students perceptions", Journal of Mechanical Design, Vol. 129 No. 7, pp. 761-768. https://doi.org/10.1115/1.2739569

Kenett, Y.N., Anaki, D. and Faust, M. (2014), "Investigating the structure of semantic networks in low and high creative persons", Frontiers in Human Neuroscience, Vol. 8, pp. 407-423. https://doi.org/10.3389/fnhum.2014.00407

Kittay, E. and Lehrer, A. (1981), "Semantic fields and the structure of metaphor", Studies in Language, Vol. 5 No. 1, pp. 31-63. https://doi.org/10.1075/sl.5.1.03kit

McClave, J.T. and Benson, P.G. (1988), Statistics for business and economy, $4^{\text {th }}$ Edition, Dellen Publishing Company, San Francisco. 
Mednick, S. (1962), "The associative basis of the creative process”, Psychological Review, Vol. 69 No. 3, pp. 220232. https://doi.org/10.1037/h0048850

Rossman, E. and Fink, A. (2010), "Do creative people use shorter associative pathways?", Personality and Individual Differences, Vol. 49 No. 8, pp. 891-895. https://doi.org/10.1016/j.paid.2010.07.025

Scott, G., Leritz, L.E. and Mumford, M.D. (2004), "The effectiveness of creativity training: a quantitative review", Creativity Research Journal, Vol. 16 No. 4, pp. 361-388. https://doi.org/10.1080/10400410409534549

Stouffer, W.B., Russel, J.S. and Oliva, M. (2004), "Making the strange familiar: creativity and the future of engineering education", Proceedings of the 2004 American Society for Engineering Education Annual Conference \& Exposition, Salt Lake City, Utah, USA, June 20-23, 2004, American Society for Engineering Education (ASEE).

Tomasco, S. (2010), IBM 2010 Global CEO Study: Creativity selected as most crucial factor for future success. [online] IBM. Available at: https:/www-03.ibm.com/press/us/en/pressrelease/31670.wss (accessed 09.12.2017)

Dr. Alejandra Beghelli, Faculty

Universidad Adolfo Ibañez, Faculty of Engineering and Sciences

Av. Padre Hurtado 750, 2340000 Viña del Mar, Chile

Email: alejandra.beghelli@uai.cl 